\title{
Disease Modifying Therapies for Transthyretin Amyloid Cardiomyopathy
}

\author{
Ariane Vieira Scarlatelli Macedo, ${ }^{1,2}$ Fábio Fernandes, ${ }^{3}$ Renato Delascio Lopes, ${ }^{1,4}$ \\ Brazilian Clinical Research Institute (BCRI), ${ }^{1}$ São Paulo, SP - Brazil \\ Santa Casa de São Paulo, ${ }^{2}$ São Paulo, SP - Brazil \\ Instituto do Coração da Faculdade de Medicina da USP, ${ }^{3}$ São Paulo, SP - Brazil \\ Duke Clinical Research Institute, Duke University Medical Center, ${ }^{4}$ Durham, NC - USA
}

Transthyretin (TTR) is a tetrameric protein synthesized essentially by the liver. TTR molecules may misfold and store as amyloid fibrils in the heart and other organs, leading to TTR-related amyloidosis (ATTR) ${ }^{1}$. ATTR can follow the deposition of either variant ATTR (ATTRv), which was previously known as mutant ATTR, or wild-type ATTR (ATTRwt). ${ }^{1}$ ATTR is an underdiagnosed disease, as well as a crucial determinant of morbidity and mortality. ${ }^{2}$ Cardiac involvement is common in ATTR, leading to transthyretin cardiomyopathy (ATTR-CA). ATTR-CA is a progressive disorder, and patients ultimately develop heart failure, arrhythmias, and cardiac conduction disturbances, resulting in decreased functional capacity, diminished quality of life, and death. ${ }^{3}$ Until recently, treatment of ATTR-CA was limited to treating symptoms and complications; however, the development of new specific therapies that delay or stop the progress of the cardiomyopathy has positively modified the outcomes ${ }^{4}$. These specific treatments work by targeting the inhibition of TTR synthesis (inotersen or patisiran); tetramer stabilization (diflunisal, tafamidis, or acoramidis [AG10]); inhibition of oligomer aggregation and disruption (epigallocatechin-3-gallate); and degradation and reabsorption of amyloid fibers (doxycycline-tauroursodeoxycholic acid [TUDCA] or doxycycline-ursodeoxycholic acid [UDCA]). 4

In patients with ATTR-CA, either ATTRv or ATTRwt, tafamidis is considered the agent of choice due to the proven clinical benefit in cardiovascular outcomes. ${ }^{5}$ Tafamidis is a kinetic TTR stabilizer that binds to the unoccupied thyroxine binding sites of tetrameric TTR and blocks the amyloidogenic cascade. ${ }^{6}$ It was first adopted for treatment of symptomatic ATTRv polyneuropathy, and it is the only therapy approved with effectiveness to treat ATTR-CA. ${ }^{5}$ This recommendation is essentially based on the results of a large, well-designed phase III randomized clinical trial (RCT), ATTR-ACT. ${ }^{7}$ In this study, tafamidis (20 and $80 \mathrm{mg}$, pooled) showed a decline in all-cause mortality and cardiovascular-related hospitalization, decreased

\section{Keywords}

Amyloidosis; Cardiac amyloidosis; Treatment; TTR; Transthyretin

Mailing Address: Renato D. Lopes •

Duke Clinical Research Institute. P.O. Box 17969, Durham, NC 27715 - USA E-mail: renato.lopes@duke.edu

Manuscript received October 12, 2021, revised manuscript October 29, 2021, accepted November 01, 2021.

DOI: https://doi.org/10.36660/abchf.20210034 decline in 6-minute walk test, slower deterioration in quality of life, and a minor increase of NT-proBNP in patients with biopsyproven ATTRwt or ATTRv CA with heart failure and NYHA class I to III at 30 months. The safety profile was similar to placebo, and individuals with NYHA classes I and II reached the most meaningful benefit. ${ }^{7}$ ATTR-ACT was not designed to assess a specific dose; therefore, further analysis from ATTR-ACT and its long-term extension study support tafamidis $80 \mathrm{mg}$ as the optimal dose to be used in clinical practice. ${ }^{8}$

Other drugs with the capacity to stabilization of TTR have been tested, but, differently from tafamidis, they have not been approved for ATTR-CA treatment yet. ${ }^{5}$ Diflunisal is a nonsteroidal anti-inflammatory (NSAID) drug with TTR-stabilizing attributes. Studies on diflunisal are all noncomparative, small non-RCTs, including almost exclusively patients with ATTRv, with exploratory cardiovascular endpoints limited to cardiovascular biomarkers and echocardiographic parameters. ${ }^{9}$ Although these potential beneficial effects have been described, adverse events associated with NSAIDs may impede their use in patients with heart failure. ${ }^{3}$

AG10 is a highly selective, small-molecule TTR stabilizer. Phase I and II studies exhibited a good toxicity profile and stabilization of both types of TTR. ${ }^{10}$ AG10 cannot be supported at present for ATTR-CA, as the only study of AG10 had a short duration of 1 month, and the cardiovascular endpoints are only exploratory and restricted to cardiac biomarkers. ${ }^{10} \mathrm{~A}$ phase III study to evaluate AG10 compared with placebo in subjects with symptomatic ATTR-CA is ongoing. The ATTRIBUTE-CM study evaluates AG10 in patients with ATTRwt or ATTRv CA, and the primary endpoints are 6-minute walk test change from baseline at 12 months and allcause mortality and cardiovascular hospitalizations at 30 months (ClinicalTrials.gov Identifier: NCT03860935).

Lately, agents able to silence the TTR gene and considerably reduce the concentration of circulating TTR have joined clinical practice. ${ }^{3}$ They specifically degrade TTR mRNA at the nucleus (inotersen) or cytoplasm (patisiran). ${ }^{11,12}$

Both agents have been accepted for the therapy of ATTRV polyneuropathy after proof of their effectiveness in neurologic RCTs. Patisiran is a promising drug for patients with ATTR-CA based on a subgroup analysis of a phase III double-blind RCT, APOLLO, ${ }^{11,13,14}$ which suggested that therapy with patisiran was associated with a decline in mortality and hospitalization, as well as a decrease in NT-proBNP and left ventricular wall thickness, without significant adverse events. By contrast, inotersen failed to demonstrate significant differences in echocardiographic variables compared with placebo in patients with ATTRv and cardiac disease in the neurological trial. ${ }^{12}$ However, a small, open-label study showed stabilization 
of cardiac parameters in most patients with ATTRv or ATTRwt followed for up to 3 years. ${ }^{15,16}$

The possible benefit of patisiran in patients with ATTR-CA is currently under research in the APOLLO-B phase III RCT. APOLLO B (ClinicalTrials.gov Identifier: NCT03997383) is assessing patisiran in patients with ATTRCA (ATTRwt or ATTRv), considering a 6-minute walk test as the primary endpoint and death and hospitalization outcomes as secondary endpoints at 12 months. Phase III trials with new gene silencing molecules are ongoing. The HELIOS-B (ClinicalTrials.gov Identifier: NCT04153149) study evaluates the RNA interference agent, vutrisiran, with the convenience of a subcutaneous administration every 3 months in patients with either ATTRwt or ATTRv CA. The primary endpoint is the composite outcome of all-cause mortality and recurrent cardiovascular hospitalizations at 30 months. The CARDIO-TTRansform trial (ClinicalTrials. gov Identifier: NCT04136171) evaluates different antisense oligonucleotides, AKCEA-TTR-LRX, with the comfort of a lower frequency of administration (every 4 weeks). This study will also include patients with ATTRwt or ATTRv CA, and the primary endpoint is cardiovascular mortality and clinical events at 120 weeks.

Another potential alternative to mRNA targeting-based gene silencing is use of the clustered regularly interspaced short palindromic repeats and associated Cas 9 endonuclease (CRISPR-Cas9) system to achieve in vivo gene editing. NTLA-2001 is a new CRISPR-Cas9-based in vivo gene-editing therapy, administered by intravenous infusion, that is intended to edit TTR in hepatocytes, leading to a decrease in the production of both wild-type and mutant TTR after a single administration. ${ }^{17}$ In patients with ATTRv with polyneuropathy, treatment with NTLA-2001 was associated with sustained reductions in the serum TTR protein concentration at day $28(87 \%$ in the group that received a dose of $0.3 \mathrm{mg}$ per kilogram). ${ }^{17}$

Additional molecules are under examination to inhibit oligomer aggregation and disruption (epigallocatechin-3gallate), accelerate clearance, and eliminate existing cardiac deposits (TUDCA). Although a small, but significant benefit was found on cardiac magnetic resonance parameters such as left ventricular mass and native T1 in patients with ATTR-CA treated with epigallocatechin-3-gallate (green tea extract), these effects should be assessed thoroughly, as they are based on noncomparative single-arm small non-RCTs. ${ }^{18-20}$

The compound of doxycycline and tauroursodeoxycholic bile acid (TUDCA) has been investigated in phase II trials, with variable outcomes and an adverse side-effect profile. ${ }^{21,22}$ At this stage, the absence of evidence and the presence of side-effects do not allow its utilization in clinical practice.
A phase III clinical trial evaluating doxycycline and TUDCA in ATTR-CM is also ongoing and will shed light on their utility (ClinicalTrials.gov Identifier: NCT03481972).

Finally, other early-phase investigations are ongoing with monoclonal antibodies that promote the removal of deposits (ClinicalTrials.gov Identifiers: NCT03336580 and NCT04360434).

Notwithstanding the tremendous ongoing investigation, there is still a data gap concerning subsets of patients who have been excluded from the studies, such as patients manifesting ATTR-CA after liver transplantation, patients with ATTR-CA with advanced heart failure (NYHA class IV), and patients with ATTR over 90 years of age. Furthermore, it is necessary to assess whether the association of treatments with distinct mechanisms of action could improve the outcome of ATTR-CA. ${ }^{5}$

As demonstrated, this is a growing research field with many RCTs currently ongoing, and their results will likely shift the paradigm of treatment of ATTR-CA. Our main goal continues to be finding the best therapy or combination of therapies for each patient with ATTR-CA by optimizing the efficacy and minimizing the side effects.

\section{Author contributions}

Conception and design of the research; Acquisition of data; Analysis and interpretation of the data; Writing of the manuscript: Macedo AVS, Fernandes F, Lopes RD. Critical revision of the manuscript for intellectual content: Macedo AVS, Fernandes F, Lopes RD.

\section{Potential Conflict of Interest}

Dra. Ariane Vieira Scarlatelli Macedo - Speaker fees for Pfizer, Novartis, Bayer, Ferring, Astra Zeneca, Janssen. Dr. Fábio Fernandes - Speaker fees for Pfizer e Alnylan. Dr. Renato D. Lopes - No potential conflict of interest.

\section{Sources of Funding}

There were no external funding sources for this study.

\section{Study Association}

This study is not associated with any thesis or dissertation work.

\section{Ethics approval and consent to participate}

This article does not contain any studies with human participants or animals performed by any of the authors. 


\section{References}

1. Saelices L, Johnson LM, Liang WY, Sawaya MR, Cascio D, Ruchala P, et al. Uncovering the Mechanism of Aggregation of Human Transthyretin. J Biol Chem. 2015;290(48):28932-43. doi: 10.1074/jbc.M115.659912.

2. Maurer MS, Bokhari S, Damy T, Dorbala S, Drachman BM, Fontana M, et al. Expert Consensus Recommendations for the Suspicion and Diagnosis of Transthyretin Cardiac Amyloidosis. Circ Heart Fail. 2019;12(9):e006075. doi: 10.1161/CIRCHEARTFAILURE.119.006075.

3. Garcia-Pavia P, Rapezzi C, Adler Y, Arad M, Basso C, Brucato A, et al. Diagnosis and Treatment of Cardiac Amyloidosis: A Position Statement of the ESC Working Group on Myocardial and Pericardial Diseases. Eur Heart J. 2021;42(16):1554-68. doi: 10.1093/eurheartj/ehab072.

4. Simões MV, Fernandes F, Marcondes-Braga FG, Scheinberg P, Correia EB, Rohde LEP, et al. Position Statement on Diagnosis and Treatment of Cardiac Amyloidosis - 2021. Arq. Bras. Cardiol. 2021;117(3):561-98. doi: $10.36660 /$ abc. 20210718.

5. Marques N, Azevedo O, Almeida AR, Bento D, Cruz I, Correia E, et al. Specific Therapy for Transthyretin Cardiac Amyloidosis: A Systematic Literature Review and Evidence-Based Recommendations. J Am Heart Assoc. 2020;9(19):e016614. doi: 10.1161/JAHA.120.016614.

6. Miroy GJ, Lai Z, Lashuel HA, Peterson SA, Strang C, Kelly JW. Inhibiting Transthyretin Amyloid Fibril Formation via Protein Stabilization. Proc Nat Acad Sci U S A. 1996;93(26):15051-6. doi: 10.1073/pnas.93.26.15051.

7. MaurerMS, SchwartzJH, Gundapaneni B, ElliottPM, Merlini G, WaddingtonCruz M, et al. Tafamidis Treatment for Patients with Transthyretin Amyloid Cardiomyopathy. N Engl J Med. 2018;379(11):1007-16. doi: 10.1056/ NEJMoa1805689.

8. Damy T, Garcia-Pavia P, Hanna M, Judge DP, Merlini G, Gundapaneni B, et al. Efficacy and Safety of Tafamidis Doses in the Tafamidis in Transthyretin Cardiomyopathy Clinical Trial (ATTR-ACT) and Long-Term Extension Study. Eur J Heart Fail. 2021;23(2):277-85. doi: 10.1002/ejhf.2027.

9. Sekijima Y, Tojo K, Morita H, Koyama J, Ikeda S. Safety and Efficacy of Long-Term Diflunisal Administration in Hereditary Transthyretin (ATTR) Amyloidosis. Amyloid. 2015;22(2):79-83. doi: 10.3109/13506129.2014.997872.

10. Judge DP, Heitner SB, Falk RH, Maurer MS, Shah SJ, Witteles RM, et al. Transthyretin Stabilization by AG10 in Symptomatic Transthyretin Amyloid Cardiomyopathy. J Am Coll Cardiol. 2019;74(3):285-95. doi: 10.1016/j. jacc.2019.03.012

11. Adams D, Gonzalez-Duarte A, O'Riordan WD, Yang CC, Ueda M, Kristen AV, et al. Patisiran, an RNAi Therapeutic, for Hereditary Transthyretin Amyloidosis. N Engl J Med. 2018;379(1):11-21. doi: 10.1056/ NEJMoa1716153.
12. Benson MD, Waddington-Cruz M, BerkJL, Polydefkis M, Dyck PJ, Wang AK, et al. Inotersen Treatment for Patients with Hereditary Transthyretin Amyloidosis. N Engl J Med. 2018;379(1):22-31. doi: 10.1056/NEJMoa1716793.

13. Solomon SD, Adams D, Kristen A, Grogan M, González-Duarte A, Maurer MS, et al. Effects of Patisiran, an RNA Interference Therapeutic, on Cardiac Parameters in Patients With Hereditary Transthyretin-Mediated Amyloidosis. Circulation. 2019;139(4):431-43. doi: 10.1161/CIRCULATIONAHA.118.035831.

14. Minamisawa M, Claggett B, Adams D, Kristen AV, Merlini G, Slama MS et al. Association of Patisiran, an RNA Interference Therapeutic, With Regional Left Ventricular Myocardial Strain in Hereditary Transthyretin Amyloidosis: The APOLLO Study. JAMA Cardiol. 2019;4(5):466-72. doi: 10.1001/jamacardio.2019.0849.

15. Benson MD, Dasgupta NR, Rissing SM, Smith J, Feigenbaum H. Safety and Efficacy of a TTR Specific Antisense Oligonucleotide in Patients with Transthyretin Amyloid Cardiomyopathy. Amyloid. 2017;24(4):219-25. doi: 10.1080/13506129.2017.1374946

16. Dasgupta NR, Rissing SM, Smith J, Jung J, Benson MD. Inotersen Therapy of Transthyretin Amyloid Cardiomyopathy. Amyloid. 2020;27(1):52-8. doi: 10.1080/13506129.2019.1685487

17. Gillmore JD, Gane E, Taubel J, Kao J, Fontana M, Maitland ML, et al. CRISPRCas9 In Vivo Gene Editing for Transthyretin Amyloidosis. N Engl J Med. 2021;385(6):493-502. doi: 10.1056/NEJMoa2107454.

18. Kristen AV, Lehrke S, Buss S, Mereles D, Steen H, Ehlermann P, et al. Green Tea Halts Progression of Cardiac Transthyretin Amyloidosis: An Observational Report. Clin Res Cardiol. 2012;101(10):805-13. doi: 10.1007/s00392-012-0463-z.

19. Aus Dem Siepen F, Buss SJ, Andre F, Seitz S, Giannitsis E, Steen H, et al Extracellular Remodeling in Patients with Wild-Type Amyloidosis Consuming Epigallocatechin-3-Gallate: Preliminary Results of T1 Mapping by Cardiac Magnetic Resonance Imaging in a Small Single Center Study. Clin Res Cardiol. 2015;104(8):640-7. doi: 10.1007/s00392-015-0826-3.

20. Aus Dem Siepen F, Bauer R, Aurich M, Buss SJ, Steen H, Altland K, et al Green Tea Extract as a Treatment for Patients with Wild-Type Transthyretin Amyloidosis: An Observational Study. Drug Des Devel Ther. 2015;9:631925. doi: 10.2147/DDDT.S96893.

21. Obici L, Cortese A, Lozza A, Lucchetti J, Gobbi M, Palladini G, et al. Doxycycline Plus Tauroursodeoxycholic Acid for Transthyretin Amyloidosis: A Phase II Study. Amyloid. 2012;19(Suppl 1):34-6. doi: 10.3109/13506129.2012.678508.

22. Wixner J, Pilebro B, Lundgren HE, Olsson M, Anan I. Effect of Doxycycline and Ursodeoxycholic Acid on Transthyretin Amyloidosis. Amyloid. 2017;24(Suppl 1):78-9. doi: 10.1080/13506129.2016.1269739. 